\title{
Study of the Very Slow Speed Solar Wind Streams with Cosmic Ray Intensity and Ae Index for Solar Cycle 24 (2008-2013)
}

\author{
Meena Pokharia and Lalan Prasad \\ Department of Physics, Govt. P.G. College, Berinag- 262531 Pithoragarh, India \\ E-mail-meenapokharia21@gmail.com
}

\begin{abstract}
The aim of this paper is to investigate the association of the variation of very slow speed solar wind streams (VSSSWS) with the cosmic ray intensity (CRI) and Ae index for solar cycle 24 (2008-2013). A Chree analysis by the superposed epoch method has been done in the study. The results of the present analysis showed that VSSSWS are not able to produce decreases in CRI. The prime source of the variation in magnetic activity near aurora zone is the wind interaction with the magnetosphere, but the speed of VSSSWS is low enough to produce any significant impact on aurora zone magnetic activity.
\end{abstract}

Keywords: Cosmic ray; Very slow speed solar wind streams; Coronal mass ejections (CMEs) .

\section{Introduction}

Solar wind is a flow of charged particles discharged from the topmost atmosphere of the Sun known as corona. Slow solar wind and fast solar wind are the two major components of solar wind in which it can be categorized. The high speed solar wind stream (HSSWS) are found to be effacious in bringing forth immense transient decrease in CRI (Shrivastava and Jaiswal, 2003). HSSWS produces transient decrease in CRI and enhancement of geomagnetic activity (Hatton, 1980). The solar wind classification procedure is described in detail by (Cliver et al., 2000). Generally, the HSSWS occurs when coronal mass ejections (CMEs) arrive. CMEs brings an explosion of highly energetic charged particles which can increase the speed of wind suddenly up to 500 $\mathrm{km} / \mathrm{sec}$ to even $1000 \mathrm{~km} / \mathrm{sec}$. But it doesn't mean that lower speed solar wind streams can't cause geomagnetic storms (GMs). If interplanetary magnetic field (IMF) conditions are good enough then, even slow speed streams can cause GMs. Cosmic ray intensity (CRI) and geomagnetic activity are two different phenomena which are affected by various solar and interplanetary parameters. Hence it is important to study the CRI in relation with various geomagnetic conditions. Akasofu et al., 1981; Troshichev et al., 2002; Verbanac et al., 2011; Kilcik et al., 2017 found that Ap and Ae shows the good relation with solar wind parameters.

\section{Data analysis and method}

A Chree analysis (Chree, 1913) by the superposed epoch method has been done in the present study with the occurrence day of VSSSWS (criteria $\mathrm{V}_{\mathrm{sw}} \leq 300 \mathrm{~km} / \mathrm{sec}$ ) as zero 
days. The pressure corrected (daily mean) CRI data has been taken from Moscow Neutron Monitor Station for solar cycle 24. The daily mean values of solar wind speed and Ae index are taken from the omni web data center (http://omniweb.gsfc.nasa.gov/cgi/nx1.cgi).

\section{Results and Discussion}

We have analyzed Fig. 1 to understand that how Ae index and CRI varies with the VSSSWS and found that for the years 2008 and 2009 the minimum peak of VSSSW and Ae coincide with zero day(0 day) which shows that as Ae decreases, VSSSW also decreases up to 0 day. While for other years, except 2008 and 2009 there exist a time lag between the minimum peak of VSSSW and Ae index. The average correlation coefficient of VSSSW with Ae and CRI is found to be high and moderate respectively. We have also compared the profile of VSSSWS with the CRI profile (Fig 1) and found that almost in every year, both these parameters are inversely related to each other (Pokharia et al., 2017).

\section{Conclusions}

The following conclusions are drawn on the basis of analysis:

1. The average correlation coefficient between VSSSW and Ae index was found 0.61 for solar cycle -24 .

2. Except for the year 2008 and 2009, there exists a time lag between the minimum peak of Ae and VSSSW.

\section{Acknowledgements}

Authors are thankful to IUCAA, Pune for content page service and also thankful to ARIES, Nainital for providing library and computing facilities.

\section{References}

Akasofu, S. I. 1981, JGR, 86 (A6), 4820-4822.

Cliver, E. W., Cane, H. V., Richardson, I. G. 2000, J. Geophys. Res., 105, 18, 203.

Chree, C., 1913, Phil. Trans. Roy. Soc., 75, A212.

Hatton, C.J.: 1980, Solar Physics. 66, 159-165.

Kilcik, A., Yiğit, E., Yurchyshyn, V., Ozguc, A., Rozelot, J. P. 2017, Sun and Geosphere,12(1), 3139.

Pokharia, M., Prasad, L., Mathpal, C., Bhoj, C., and Mathpal, R. 2017, Journal of Pure Applied and Industrial Physics, 7 (5), 162-165.

Shrivastava, P.K., Jaiswal, K.L.: 2003, Sol. Phys. 214, 195.

Troshichev, O.A., Lukianova, R. Yu. 2002, Journal of Atmospheric and Solar-Terrestrial Physics, 64, 585-591

Verbanac, G., Vrsnak, B., Zivkovi, S., Hojsak, T., Veronig, A. M., and Temmer, M. 2011, A\&A, 533 (A49). 


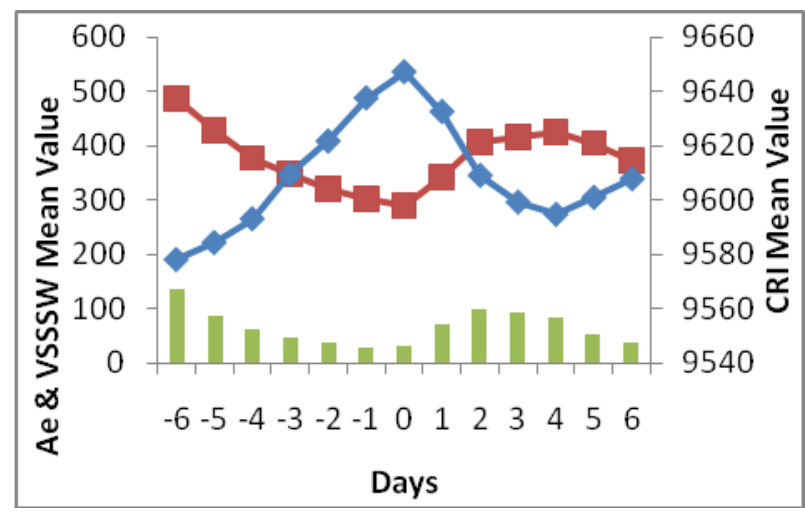

2008

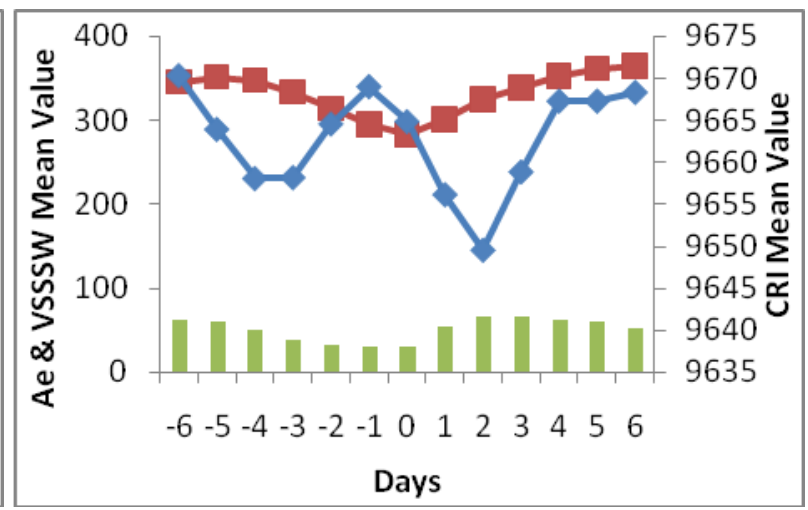

2009

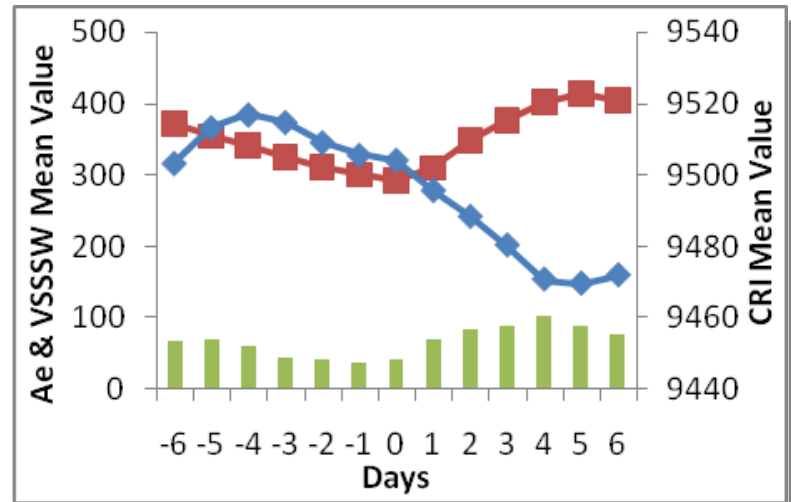

2010

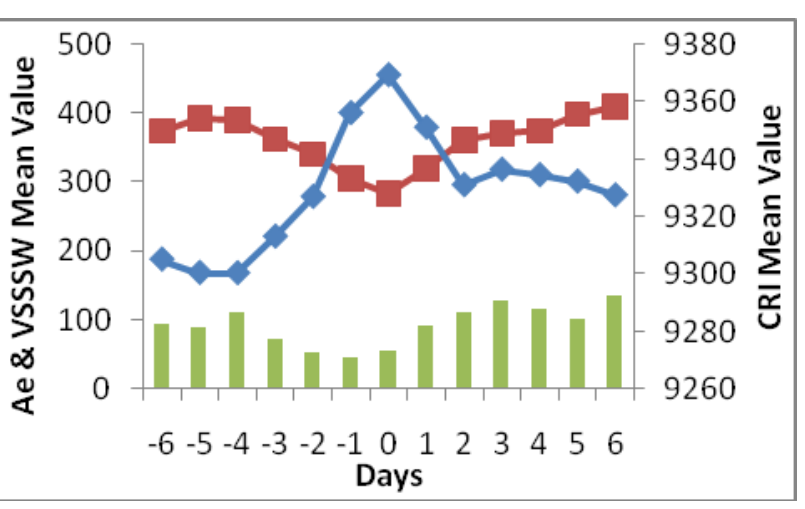

2011

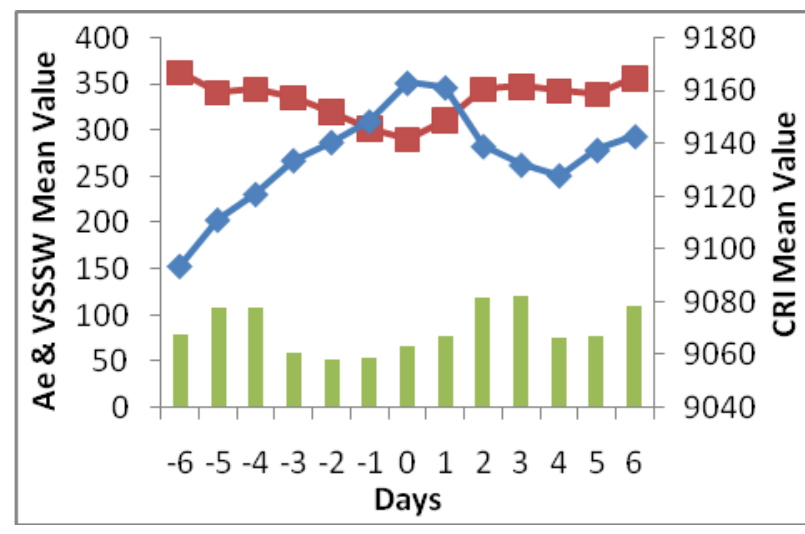

2012

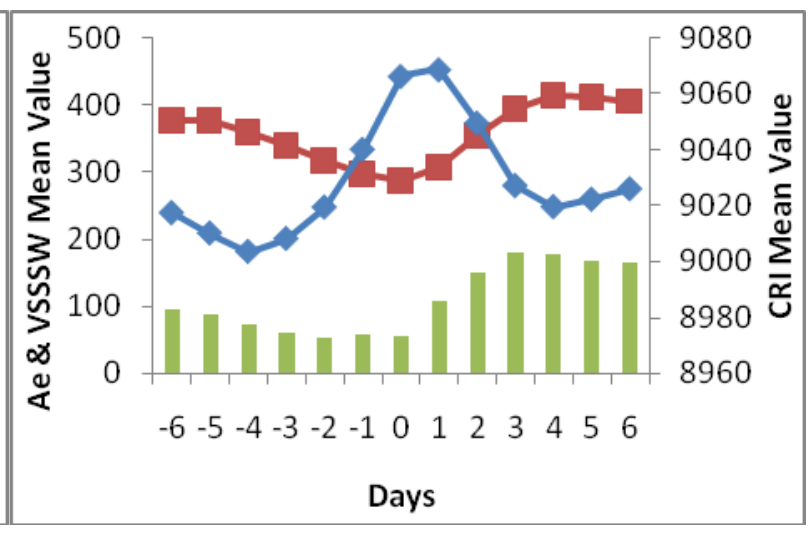

2013

\section{Ae index $\rightarrow$ CRI - VSSSW}

Figure 1(Color online): The result of Chree analysis from -6 day to +6 days with respect to zero epoch day (occurrence of VSSSWS) 